\title{
Viability of five different pre- and intraoperative imaging methods for autologous breast reconstruction
}

\author{
K. F. Schrögendorfer · S. Nickl · M. Keck · D. B. Lumenta · C. Loewe - M. Gschwandtner · W. Haslik · \\ J. Nedomansky
}

Received: 8 August 2016 / Accepted: 14 September 2016 / Published online: 19 October 2016 (C) The Author(s) 2016. This article is available at SpringerLink with Open Access.

\begin{abstract}
Summary
Background Autologous breast reconstruction is an integral part in the treatment of breast cancer. While computed tomography angiography (CTA) is an established preoperative diagnostic tool for microsurgeons, no study has so far evaluated and compared five different imaging methods and their value for the reconstructive team. In order to determine the feasibility of each of the tools for routine or specialized diagnostic application, the methods' efficiency and informative value were analyzed.

Methods We retrospectively analyzed imaging data of 41 patients used for perforator location and assessment for regional perfusion and vessel patency in patients undergoing autologous breast reconstruction with deep inferior epigastric perforator flap (DIEP), transverse rectus abdominis muscle flap (TRAM), or transverse myocutaneous gracilis flap (TMG). Five different imaging techniques were used: hand held Doppler (HHD), CT angiography (CTA), macroscopic indocyanine green (ICG) video angiography, microscope-integrated ICG video angiography, and laser Doppler imaging (LDI).
\end{abstract}

K. F. Schrögendorfer, M.D. · S. Nickl, M.D. · M. Keck, M.D.

D. B. Lumenta, M.D. · W. Haslik, M.D. ( $\square)$.

J. Nedomansky, M.D.

Division of Plastic and Reconstructive Surgery, Department of Surgery, Medical University of Vienna, Währinger Guertel 18-20, 1090 Vienna, Austria

werner.haslik@meduniwien.ac.at

C. Loewe, M.D.

Department of Radiology, Division of Cardiovascular and Interventional Radiology, Medical University of Vienna, Vienna, Austria

M. Gschwandtner, M.D.

Department of Angiology, Medical University of Vienna, Vienna, Austria
Results CTA proved to be the best tool for preoperative determination of the highly variable anatomy of the abdominal region, whereas HHD showed the same information on perforator localization with some falsepositive results. Intraoperative HHD was an excellent tool for dissection and vessel patency judgment. Microscope-integrated ICG was an excellent tool to document the patency of microanastomoses. In our series, macroscopic perfusion measurement with ICG or LDI was only justified in special situations, where information on perfusion of abdominal or mastectomy flaps was required. LDI did not add any additional information.

Conclusion Preoperative assessment should be performed by CTA with verification of the perforator location by HHD. Intraoperative HHD and microscopeintegrated ICG contribute most toward the evaluation of vessel patency. ICG and LDI should only be used for special indications.

Keywords Autologous breast reconstruction - Computed tomography angiography · Laser Doppler imaging · Indocyanine green · Imaging methods

\section{Introduction}

Breast reconstruction is an integral part in the treatment of breast cancer to overcome the sequelae associated with tumor resection and radiotherapy. Several reconstructive strategies have become available, depending on the requirements for each patient. One of the main objectives is to provide a safe and reliable method to recreate an esthetically pleasing breast. When removal of all breast tissue is necessary, autologous breast reconstruction has proven to deliver optimal results in terms of shape, natural appearance, and pliability [1-5]. However, autologous breast reconstruction with deep inferior epigastric 
Table 1 Synopsis of all imaging methods in the different phases of breast reconstruction

\begin{tabular}{|c|c|c|c|c|c|}
\hline Phases & CTA & HHD & ICG macro & ICG micro & LDI \\
\hline Preoperative & Yes & Yes & No & No & No \\
\hline Intraoperative before microanastomoses & No & Yes & Yes & No & Yes \\
\hline Intraoperative after microanastomoses & No & Yes & No & Yes & Yes \\
\hline
\end{tabular}

perforator flap (DIEP), transverse rectus abdominis muscle flap (TRAM), or transverse myocutaneous gracilis flap (TMG) is associated with longer operation time, advanced surgical technique involving microsurgery, and an increased risk of flap loss compared with implants or pedicled myocutaneous latissimus dorsi flaps (MLD) [6]. Partial or complete flap loss in this reconstructive setting is always a catastrophe and must be avoided at all costs with all precautions taken. Therefore, many technical devices have been developed to provide the surgeon with information on the localization of perforators, patency of vessels or microanastomoses, and perfusion of tissue with the primary aim of minimizing complications and optimizing outcome. All of these techniques have been described in the literature to varying extent [7-15]. However, the efficiency of different diagnostic methods and their redundant information with respect to each stage in microsurgical breast reconstruction have not been weighed against each other in a summarized context.

One approach to comparing different diagnostic measurements is to analyze each of them based on distinct parameters such as interpretive value, time efficiency, associated risks, availability, and costs.

In general, diagnostic measurements can be differentiated into the three distinct phases of microsurgical breast reconstruction: preoperatively, intraoperatively before microsurgery, and intraoperatively after microsurgery. In order to determine the feasibility of each of the tools for routine or specialized diagnostic application, we analyzed each of the methods' efficiency and informative value for the surgeon during the aforementioned phases.

\section{Materials and methods}

We retrospectively analyzed imaging data used for perforator location and patency as well as for assessment of regional perfusion and vessel patency in all patients undergoing autologous breast reconstruction with DIEP, TRAM, or TMG in our institution during a 3-year period. This study was approved by the local ethics committee (EK 184/2011).

Five different imaging techniques were used: handheld Doppler (HHD), computed tomography angiography (CTA), macroscopic indocyanine green (ICG) video angiography, microscope-integrated ICG video angiography (mi ICG), and laser Doppler imaging (LDI). These methods were used and applied in all or several of the three different stages of breast reconstruction (Table 1).

HHD (bidirectional Doppler probe, Multi Dopplex II, Huntleigh Technology Ltd., UK) was used for the detection of the perforator location and the assessment of its flow. Preoperatively, the abdominal donor site was examined in order to localize perforators, and the appropriate sites were marked with a waterproof ink marker. Intraoperatively, HHD was used to confirm the integrity of the dissected perforators before microsurgery, and to judge vessel patency after microsurgery.

CTA (Siemens Somatom Definition Flash using $120 \mathrm{kV}$ and dose adapted mAs) with intravenously applied contrast agent $\left(90 \mathrm{ml}\right.$ of Iomeron ${ }^{\circledR} 400 \mathrm{mg} / \mathrm{ml}$, Bracco, Austria; with an injection speed of $5 \mathrm{ml} / \mathrm{s}$ followed by a saline flush of $40 \mathrm{ml}$ ) was performed preoperatively to assess the vessel, muscle, soft tissue, and intra-abdominal anatomy of the abdominal donor region. For improved visualization, the acquired images were reconstructed using volume-rendered and maximum intensity projection techniques. The number and diameter of perforators were determined. The course of the deep inferior epigastric vessels and their branches in relation to the rectus abdominis muscle as well as related morphology (e.g., rectus diastasis) were evaluated. A transparent grid was used to allow for optimal projection of all detected data in relation to the umbilicus on a data sheet, and was marked on the abdominal donor region with an ink marker on the day before surgery. Intraoperative findings were defined as the standard, and HHD vs. CTA was compared with these findings.

Macroscopic ICG angiography is a near-infrared technique of dynamic laser fluorescence videography $\left(\mathrm{IC}-\mathrm{View}^{\circledR}\right.$, Pulsion Medical Systems, Munich, Germany) [16] for assessment of tissue perfusion. It was only used in cases where large tissue harvests were necessary in order to visualize blood flow and estimate the maximal possible size of the raised abdominal tissue allowing for sufficient flap perfusion. After cleaning the region of interest (ROI) from blood [17], a dose of $0.2 \mathrm{mg} / \mathrm{kg}$ ICG was administered intravenously. The perfusion dynamics were visualized in real time and simultaneously stored in the system for subsequent analysis (IC-Calc ${ }^{\circledR}$; Pulsion Medical Systems, Munich, Germany). The skin perfusion of the raised flap in the ROI was expressed as mean pixel intensity; thereby the site of the perforator, the very 
Table 2 Detailed synopsis of imaging methods in the preoperative phase

\begin{tabular}{|c|c|c|}
\hline & Preoperative phase & \\
\hline Imaging method & CTA & $H H D$ \\
\hline Interpretive value & High, displays all relevant details & No qualitative assessment possible \\
\hline Handling & If established, no long waiting list, RTA familiar with procedure & Easy \\
\hline Redundant information & No & CTA \\
\hline Time efficiency for surgeon & Analysis can be done independent of the presence of patient & Evaluation can only be done on the patient, $45 \mathrm{~min}$ \\
\hline Time efficiency for patient & $\begin{array}{l}\text { 2nd appointment necessary, but quick investigation, and quick mark- } \\
\text { ing preoperative }\end{array}$ & Long duration of investigation, $45 \mathrm{~min}$ \\
\hline Associated risks & Radiation and iv contrast agent & No \\
\hline Availability & CTA is available in all hospitals providing breast reconstruction & Unlimited \\
\hline Value for money & Some costs, but highly effective & Cheap, but only limited information \\
\hline
\end{tabular}

edge of the flap, and the later discarded part of the flap were determined as ROIs.

Microscope-integrated ICG video angiography: Microscope-integrated ICG was performed with three different microscopes to determine the patency of the microsurgery, depending on the availability of each device: MÖLLER Hi-R 20-1000G ICG ${ }^{\circledR}$, ZEISS OPMI Pentero Infrared $800^{\circledR}$, and LEICA M720 OH5 ${ }^{\circledR}$. All systems contain a three-chip HD and a TV camera with a near-infrared spectrum. After intravenous application of $0.2 \mathrm{mg} / \mathrm{kg}$ ICG, the blood flow in the vessels was visualized and was recorded by an infrared camera and displayed on a monitor adjacent to the microscope allowing for the assessment of vessel patency.

A PIM II LDI device (Laser Doppler Perfusion Imager, Perimed, Sweden) was used in selected cases to evaluate the perfusion of abdominal flaps before and after microsurgery. The ROI was scanned by a laser beam resulting in a color-coded image of the blood perfusion.

The five methods were independently analyzed by two surgeons involved in the patients' treatment, and the quality of information was assessed using the following parameters:

- Interpretive value (false positive, false negative)

- Handling

- Redundant information

- Time efficiency for the surgeon (presence of patient required for analysis, intraoperative application, effect on overall duration of surgery)

- Time efficiency for the patient (e.g., separate appointments, waiting lists, effect on overall duration of surgery)

- Associated risks (e.g., radiation, extended duration of surgery)

- Availability (e.g., waiting lists, specialized centers etc.)

- Value for money (costs for patient/health-care system and value of information for surgeon)

Each of the parameters was judged against a rating scale, which ranged from excellent to poor, with good and fair representing intermediate evaluations (Tables 2, 3 and 4).

\section{Statistical analysis}

For descriptive statistics we used IBM ${ }^{\circledR}$ SPSS ${ }^{\circledR}$ Statistics 18 (Chicago Ill.). Values are expressed as mean and standard deviation for parametric and median (minimum-maximum) for nonparametric data.

\section{Results}

A total of 41 patients with 43 autologous breast reconstructions were treated in our hospital in the study period. Among these patients, 29 DIEP flaps (28 unilateral, 1 bilateral), five unilateral pedicled TRAM flaps, and nine TMG flaps (eight unilateral, one bilateral) were performed; 17 patients underwent immediate reconstructions, and 24 underwent secondary reconstructions. In this collective, $41 \mathrm{HHD}$, 25 CTA, eight ICG microscopic-based angiographies, five macroscopic flap ICG angiographies, and three LDI methods were performed. We did not observe any total or partial flap loss. There was no occurrence of postoperative hematoma or infection, seroma formation occurred in four patients, and one patient developed skin necrosis of the abdominal donor site after harvest of a DIEP flap.

The results of the imaging methods were stratified into three different time periods: preoperative phase, intraoperative phase pre-microsurgery, and intraoperative phase post-microsurgery. The assessment of each respective imaging method is summarized in Tables 2,3 and 4 .

\section{Preoperative phase (HHD, CTA)}

CTA was found to have a $100 \%$ sensitivity and specificity rate for perforator localization (vs. intraoperatively confirmed anatomy). Rectus diastasis was found in 23 of 25 patients during CTA (median: $2.5 \mathrm{~cm}$, $0-4.5 \mathrm{~cm}$ ). The mean number of perforators was $4.28 \pm$ 0.99 , with an average diameter of $1.73 \pm 0.47 \mathrm{~mm}$. The 
Table 3 Detailed synopsis of imaging methods in the intraoperative phase before microanastomoses

\begin{tabular}{|c|c|c|c|}
\hline & Intraoperative pre-anastomoses & & \\
\hline Imaging method & HHD & Macroscopic ICG & LDI \\
\hline Interpretive value & Adequate & Dynamic investigation & Static investigation \\
\hline Handling & Easy & Requires training & Requires training \\
\hline Redundant information & No & $L D I$ & ICG \\
\hline Time efficiency for surgeon & Quick assessment of perforators & 10 min extension of op time & 30 min extension of op time \\
\hline Time efficiency for patient & No time consuming procedure & 10 min extension of op time & 30 min extension of operating time \\
\hline Associated risks & No & I.V. application of ICG & No \\
\hline Availability & Easy available & Special equipment & Special equipment \\
\hline Value for money & $\begin{array}{l}\text { Good information, quick evaluation, } \\
\text { eases preparation }\end{array}$ & $\begin{array}{l}\text { Only if clinical judgement is in } \\
\text { doubt (selected cases) }\end{array}$ & $\begin{array}{l}\text { No additional information in compari- } \\
\text { son with ICG }\end{array}$ \\
\hline
\end{tabular}

Table 4 Detailed synopsis of imaging methods in the intraoperative phase after microanastomoses

\begin{tabular}{l|l|l|}
\hline & Post anastomoses & HHD \\
\hline Imaging method & Microscope integrated ICG & No qualitative assessment possible \\
\hline Interpretive value & Excellent display of vessel patency & Easy \\
\hline Handling & Easy & ICG micro \\
\hline Redundant information & No & Easy, but difficult to interpret (vein) \\
\hline Time efficiency for surgeon & $5 \mathrm{~min}$ & Quick procedure \\
\hline Time efficiency for patient & $5 \mathrm{~min}$ & No \\
\hline Associated risks & i.V. application of ICG & Easy available \\
\hline Availability & High costs, integrated only in new microscopes & Cheap, but no reliable information \\
\hline Value for money & Expensive, but best information about patency of microanastomoses & \\
\hline $\begin{array}{l}\text { Normal type represents "excellent" evaluation on rating scale; bold type represents "poor" evaluation on rating scale; italic type represents "good" evaluation } \\
\text { on rating scale }\end{array}$ &
\end{tabular}

scan time for CTA was $1.5 \mathrm{~s}$; the mean time for image analysis by surgeons was $15 \pm 10 \mathrm{~min}$. The transfer of important markings to the patient's surface took about 5 min.

By contrast, the HHD had $100 \%$ sensitivity and $97.1 \%$ specificity (vs. intraoperatively confirmed anatomy). Owing to the nature of HHD there was no possibility to quantify vessel diameter or to identify the anatomical course through the muscle. The time needed for the surgeon to localize and mark the perforators was 15-45 min depending on the vessel diameter, its course, as well as the patient's body weight and thickness of the subcutaneous tissue.

\section{Intraoperative phase (HHD, macroscopic ICG, LDI)}

During the intraoperative phase, HHD was repeated under sterile conditions to confirm perforator localization during flap harvest and to evaluate the perforator patency after intramuscular dissection. In this context, HHD was found to be reliable, and no falsepositive or false-negative results were noted.

The median mean pixel intensity of macroscopic ICG was 39.6 (37.6-44.8) in the area of the perforator, $31.2(29.1-38.9)$ at the end of the flaps, and 21.9 (19.4-30.1) in the discarded parts of the flaps (Figs. 1 and 2).
LDI was used in three cases as a noninvasive method for determination of the flap perfusion. In one case, flap ischemia occurred during skin closure, and LDI confirmed compromised flap perfusion before microsurgical revision.

In cases where macroscopic ICG or LDI was used, no malperfused region was detected intraoperatively, and no skin or fat necrosis occurred during postoperative follow-up.

\section{Post-microsurgery phase (microscope-integrated ICG, $H H D$ )}

Microscope-integrated ICG demonstrated vessel patency in six out of eight cases. In the remainder, no flow was shown on ICG, and resulted in the decision to perform immediate microsurgical revision $(n=2)$; HHD was performed in all cases to evaluate Doppler signaling in the distal part of the microanastomosis. Arterial acoustic detection was clearly audible in all patent anastomoses; however, venous signaling was difficult to classify, and did not allow for definitive judgment of the vessel patency. 

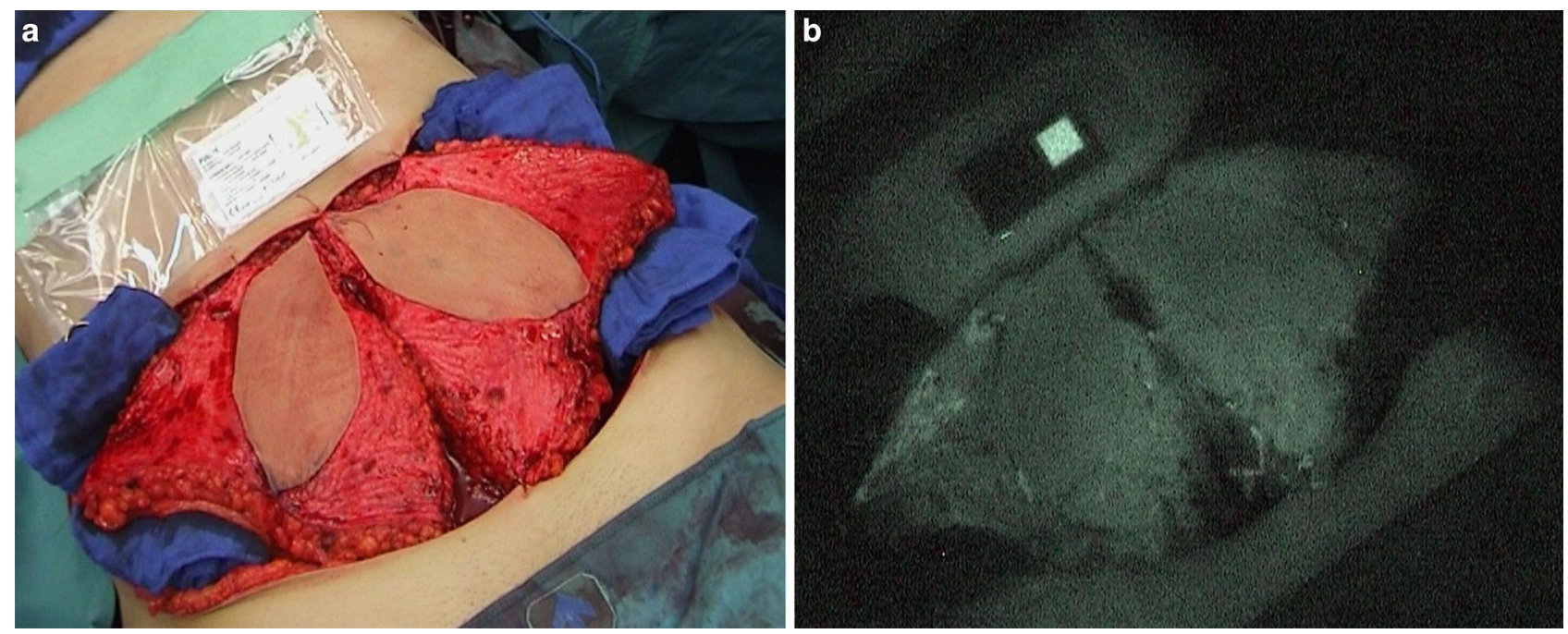

Fig. 1 a Real-time view of a bilateral DIEP before ICG video. b Steady-state ICG view after application of ICG dye. Note homogeneous perfusion of both entire flaps

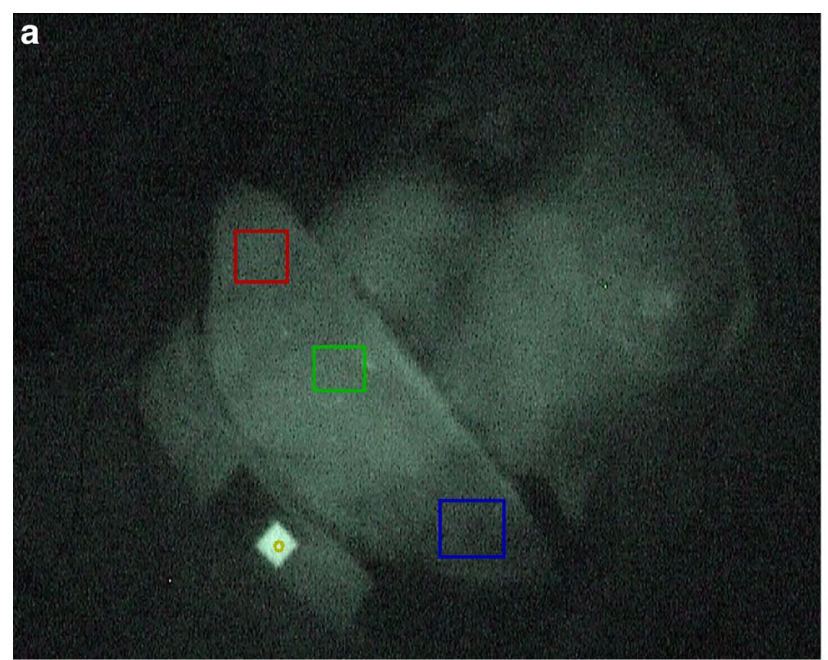

b

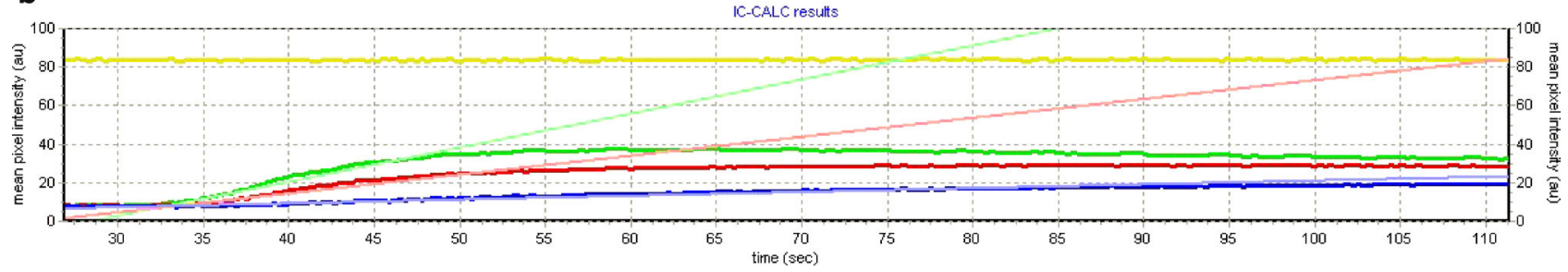

Fig. 2 a Raised DIEP flap in ICG view, steady state after ICG dye application. Green area: localization of perforator; red area: border of flap design; blue area: discarded area. b Green curve: site of perforator; red curve: edge of flap; blue curve: discarded tissue of the flap

\section{Discussion}

Autologous free flaps are an essential part of breast reconstruction, albeit technically more demanding and more time consuming than implant-related techniques or pedicled flaps. Nonetheless, their results bear the most advantages of autologous tissue reconstruction: natural feel, no follow-up surgery, and no foreign-body-related reactions.
A key element for a successful autologous breast reconstruction is identifying the entire course of the tissue supplying blood vessels. Accurate preoperative imaging is fundamental when developing a surgical plan in order to refrain from time-consuming intraoperative assessments especially in the presence of multiple perforators of similar caliber and flow [18, 19]. 


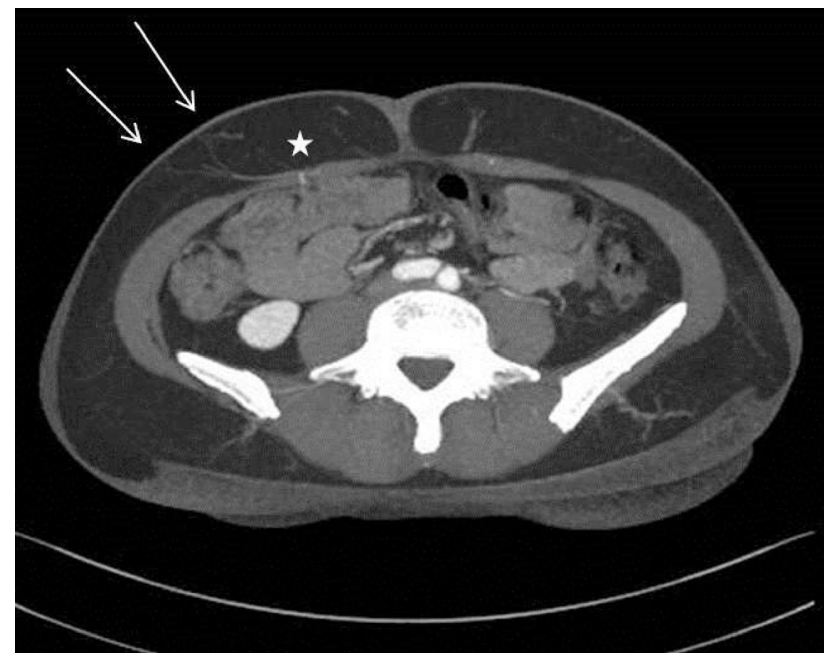

Fig. 3 MDCT picture before DIEP breast reconstruction showing differences between actual position of perforator and Doppler signal. Star indicates real position of perforator piercing the fascia. White arrow indicates the position of audible preoperative Doppler signal

In our retrospective analysis we evaluated imaging techniques whose application provided the surgical team with relevant clinical information during three operative stages. In the preoperative phase, CTA was performed on all patients undergoing abdominal flap reconstruction. CTA has proven to be a method of choice for the preoperative investigation of perforators and has aided in reducing operative time [13, 20]. It allowed for the precise demonstration of their intramuscular course, localization, and caliber. In addition, the radiation dose applied can be minimized by choosing the appropriate ROI, and was the case in our patient collective analyzed in conjunction with previous reports $[7,21]$. While examination planning and technical infrastructure require more time in the preoperative phase, the benefits of CTA have proven to offset these shortcomings (see Table 1, Preoperative). So far, magnetic resonance angiography has been evaluated in different studies, but has not yet gained the same popularity as CTA. However, this technique could be a radiation-free option for the future [22-24].

HHD is a noninvasive operator-dependent procedure, which can be applied in a variety of clinical contexts. While its associated costs are limited to its one-time purchase, it does not allow for quantification of blood flow and visualization of anatomical structures. Additionally, it required the surgeon's and patient's presence during the entire procedure, and did not permit a post hoc analysis of data in contrast to CTA. From a surgical point of view, it was more convenient to have a preoperative examination performed in a time- and location-independent setting. HHD also delivered false-positive results with regard to perforator location in comparison to CTA (Fig. 3), which was described before $[8,25]$. HHD proved to be an excellent device for validating the CTA markings preoperatively.

Intraoperatively, HHD aided in facilitating perforator dissection and evaluating vessel patency, and was hence an essential tool for the operative team.

In our study microscope-integrated ICG video angiography reliably detected vessel patency/occlusion after microsurgery leading to immediate revision. In these cases, neither flap revision was required nor partial or total flap loss was observed. However, whether this was related to the application of this method remains hypothetical in this study's context. In a report by Holm et al., ICG demonstrated $100 \%$ sensitivity and $86 \%$ specificity for the detection of microvascular thrombosis in 20 patients with clinical signs of flap malperfusion [10]. This objective method allows for the intraoperative evaluation of vessel patency, possibly reduces the requirement for intraoperative emptyand-refill tests, and facilitates immediate documentation of the microsurgical results [14].

The difference between the microscope-integrated and the macroscopic video camera-based ICG system lies essentially in the smaller field of vision through the microscope. A camera-only-based system allows for portability and variable use under different clinical circumstances. It proved to be a reliable method for performing dynamic perfusion investigations in DIEP and SIEA flaps [9]. From our point of view, its use provided an objective intraoperative dynamic evaluation of tissue perfusion, and could, for example, be applied for visualizing perfusion in post-mastectomy skin flaps during immediate breast reconstruction [26], large single perforator flaps, or bilateral DIEP flaps, if viability is in doubt.

The laser-based noninvasive perfusion measurement delivered static information, and did not require intravenous application of a contrast agent as in ICGbased methods. It is therefore an objective method for determining the final steady state of tissue perfusion in a research setting. However, its clinical setup was quite cumbersome, and in fact prolonged the operative procedure without providing more information than the aforementioned method.

\section{Conclusion}

- In our study, CTA proved to be the best tool for preoperative determination of the highly variable anatomy of the abdominal region and should be used in any patient despite cost and radiation dose.

- HHD provided the same information on perforator localization preoperatively, with some false-positive results, but it does not display the course of the vessel. Therefore, this investigation need not be done preoperatively, but can be used for confirmation of CTA markings.

- In the intraoperative setting, HHD is an indispensable, quick, reliable, and cheap tool for dissection 
and vessel patency judgment with no burden for the patient.

- Macroscopic ICG is only justified in special situations where information on the perfusion border of the abdominal flaps or mastectomy flaps is needed.

- Microscope-integrated ICG is an excellent tool to document the patency of the mircoanastomoses and can also be used to check the viability of the mastectomy flap.

- LDI proved to be a noninvasive tool for the assessment of skin perfusion; however, it does not add additional information to the other techniques and was therefore abandoned.

Acknowledgements We would like to thank Silvia Unterhumer for her formidable assistance in performing CT angiographies.

Open access funding provided by Medical University of Vienna.

Conflict of interest K.F. Schrögendorfer, S. Nickl, M. Keck, D.B. Lumenta, C. Loewe, M. Gschwandtner, W. Haslik, and J. Nedomansky declare that they have no competing interests.

Open Access This article is distributed under the terms of the Creative Commons Attribution 4.0 International License (http://creativecommons.org/licenses/by/4.0/), which permits unrestricted use, distribution, and reproduction in any medium, provided you give appropriate credit to the original author(s) and the source, provide a link to the Creative Commons license, and indicate if changes were made.

\section{References}

1. Alderman AK, Wilkins EG, Lowery JC, Kim M, Davis JA. Determinants of patient satisfaction in postmastectomy breast reconstruction. Plast Reconstr Surg. 2000;106(4):769-76.

2. Kadle R, Cohen J, Hambley W, Gomez-Viso A, Rifkin W, Allen R, et al. A 35-year evolution of free flap-based breast reconstruction at a large urban academic center. J Reconstr Microsurg. 2016;32(2):147-52.

3. Kroll SS, Baldwin B. A comparison of outcomes using three different methods of breast reconstruction. Plast Reconstr Surg. 1992;90(3):455-62.

4. Saulis AS, Mustoe TA, Fine NA. A retrospective analysis of patient satisfaction with immediate postmastectomy breast reconstruction: comparison of three common procedures. Plast Reconstr Surg. 2007;119(6):1669-78.

5. Seidenstuecker K, van Waes C, Munder BI, Claes KE, Witzel C, Roche N, et al. DIEAP flap for safe definitive autologous breast reconstruction. Breast. 2016;26:59-66.

6. Cordeiro PG. Breast reconstruction after surgery for breast cancer. NEngl J Med. 2008;359(15):1590-601.

7. Masia J, Clavero JA, Larranaga JR, Alomar X, Pons G, SerretP. Multidetector-row computed tomography in the planning of abdominal perforator flaps. J Plast Reconstr Aesthet Surg. 2006;59(6):594-9.

8. Giunta RE, Geisweid A, Feller AM. The value of preoperative Doppler sonography for planning free perforator flaps. Plast Reconstr Surg. 2000;105(7):2381-6.

9. Holm C, Mayr M, Hofter E, Becker A, Pfeiffer UJ, Muhlbauer $\mathrm{W}$. Intraoperative evaluation of skin-flap viability using laser-induced fluorescence of indocyanine green. Br J Plast Surg. 2002;55(8):635-44.

10. Holm C, Dornseifer U, Sturtz G, Ninkovic M. Sensitivity and specificity of ICG angiography in free flap reexploration. J Reconstr Microsurg. 2010;26(5):311-6.

11. Booi DI, Debats IB, Boeckx WD, van der Hulst RR. Risk factors and blood flow in the free transverse rectus abdominis (TRAM) flap: smoking and high flap weight impair the free TRAM flap microcirculation. Ann Plast Surg. 2007;59(4):364-71.

12. Klasson S, Svensson H, Malm K, Wasselius J, Velander P. Preoperative CT angiography versus Doppler ultrasound mapping of abdominal perforator in DIEP breast reconstructions: A randomized prospective study. J Plast Reconstr Aesthet Surg. 2015;68(6):782-6.

13. Fitzgerald O'Connor E, Rozen WM, Chowdhry M, Band B, Ramakrishnan VV, Griffiths M. Preoperative computed tomography angiography for planning DIEP flap breast reconstruction reduces operative time and overall complications. Gland Surg. 2016;5(2):93-8.

14. Griffiths M, Chae MP, Rozen WM. Indocyanine green-based fluorescent angiography in breast reconstruction. Gland Surg. 2016;5(2):133-49.

15. Vargas CR, Koolen PG, Ho OA, Tobias AM, Lin SJ, Lee BT. Preoperative CT-angiography in autologous breast reconstruction. Microsurgery. 2016. doi:10.1002/micr. 30065.

16. Kamolz LP, Andel H, Haslik W, Donner A, Winter W, Meissl G, et al. Indocyanine green video angiographies help to identify burns requiring operation. Burns. 2003;29(8):785-91.

17. Haslik W, Kamolz LP, Andel H, Winter W, Meissl G, Frey $M$. The influence of dressings and ointments on the qualitative and quantitative evaluation of burn wounds by ICG video-angiography: an experimental setup. Burns. 2004;30(3):232-5.

18. Schaverien M, Saint-Cyr M, Arbique G, Brown SA. Arterial and venous anatomies of the deep inferior epigastric perforator and superficial inferior epigastric artery flaps. Plast Reconstr Surg. 2008;121(6):1909-19.

19. Patel SA, Keller A. A theoretical model describing arterial flowin the DIEP flap related to number and size of perforator vessels. J Plast Reconstr Aesthet Surg. 2008;61 (11):1316-20.

20. Smit JM, Dimopoulou A, Liss AG, Zeebregts CJ, Kildal M, Whitaker IS, et al. Preoperative CT angiography reduces surgery time in perforator flap reconstruction. J Plast Reconstr AesthetSurg. 2009;62(9):1112-7.

21. Phillips TJ, Stella DL, Rozen WM, Ashton M, Taylor GI. Abdominal wall CT angiography: a detailed account of a newly established preoperative imaging technique. Radiology. 2008;249(1):32-44.

22. Rozen WM, Stella DL, Bowden J, Taylor GI, Ashton MW. Advances in the pre-operative planning of deep inferior epigastric artery perforator flaps: magnetic resonance angiography. Microsurgery. 2009;29(2):119-23.

23. Alonso-Burgos A, Garcia-Tutor E, Bastarrika G, Benito A, Dominguez PD, Zubieta JL. Preoperative planning of DIEP and SGAP flaps: preliminary experience with magnetic resonance angiography using 3 -tesla equipment and blood-pool contrast medium. J Plast Reconstr Aesthet Surg. 2010;63(2):298-304.

24. Masia J, Kosutic D, Cervelli D, Clavero JA, Monill JM, Pons G. In search of the ideal method in perforator mapping: noncontrast magnetic resonance imaging. J Reconstr Microsurg. 2010;26(1):29-35.

25. Blondeel PN, Beyens G, Verhaeghe R, Van Landuyt K, Tonnard P, Monstrey SJ, et al. Doppler flowmetry 
in the planning of perforator flaps. Br J Plast Surg. 1998;51(3):202-9.

26. Komorowska-Timek E, Gurtner GC. Intraoperative perfusion mapping with laser-assisted indocyanine green imaging can predict and prevent complications in immediate breast reconstruction. Plast Reconstr Surg. 2010;125(4):1065-73. 